\title{
Cystiysticercosis as a differential diagnosis of knee pain: A rare case report
}

\author{
Ali Mohammed P ${ }^{1}$, Jagdish Menon ${ }^{2}$, DK Patro ${ }^{3}$, Deep Sharma ${ }^{4}$ \\ ${ }^{1}$ Dr Ali Mohammed P, Senior Resident, Department of Orthopaedics, ${ }^{2}$ Dr Jagdish Menon, Head Department of \\ orthopaedics, ${ }^{3}$ Dr D K Patro Senior Professor, Department of orthopaedics, ${ }^{4}$ Dr Deep Sharma, Associate Professor, \\ Department of Orthopaedics. All are affiliated with Department of orthopaedics, Jawaharlal Institute of Postgraduate \\ Medical Education and Research Pondicherry (JIPMER), India
}

Address for Correspondence: Dr Ali Mohammed P, Email: dralinisreenmohammedp@gmail.com, No 32,Thiruvalluvar Street,Dr Radhakrishna Nagar,Pondicherry, India.

\begin{abstract}
Cysticercosis is caused by larval stage of pork tapeworm(Taenia solium). There is difficulty in diagnosing cysticercosis by ultrasound alone in its early stage especially if the lesion is small.we need to adopt MRI for early diagnosis and for starting optimum treatment for the patient. We discuss about a case of cysticercosis of vastus medialis muscle who presented with knee pain in orthopedic OPD and medical treatment was given with complete resolution of infection. Case Report: A 31 years old male who presented with complaints of pain in the (L) knee for 2 weeks duration, difficulty in sitting cross legged and squatting for 1 week. He was also complaining of mild swelling on the medial aspect of the knee. On local examination, a diffuse swelling of distal thigh (medial aspect) with knee effusion was present with induration of vastus medialis muscle. On ultrasonography it was diagnosed as a small tear in the vastus medialis muscle.But MRI scan showed evidence of $1 \times 0.8 \times 0.5 \mathrm{~cm}$ well defined oval cystic lesion in the deep muscular plane of vastus medialis in its distal one third with surrounding muscle edema and knee effusion. Patient was completely asymptomatic with full range of knee ROM after treatment with albendazole alone for 4 weeks..Conclusion: We should keep Intra muscular cysticercosis as a differential diagnosis for knee pain especially in endemic areas. Muscular cysticercosis with no other systemic involvement can be treated with albendazole alone. Since isolated cysticercosis is a rare entity we should always rule out other systemic involvement including central nervous system.
\end{abstract}

Keywords:Vastus Medialis, Cysticercosis,MRI,Knee Pain,Conservative Treatment.

\section{Introduction}

Cysticercosis is caused by larval stage of pork tapeworm (Taenia solium) [1]. Its cystic stage (cysticercus) affects various parts of human body which includes lungs, heart, peritoneum, muscles, and the maxillofacial region [2,3]. Soft tissue/muscular cysticercosis is most often associated with nervous system involvement[4-10].but there are few case reports where we have seen muscular cysticercosiswith no multiple cysts/brain involvement[5,6,8,9,11-14].

Here we are presenting a case of vastus medialis muscle involvement with no other systemic involvement who

Manuscript received:2 $4^{\text {th }}$ Sept 2015

Reviewed: $4^{\text {th }}$ Oct 2015

Author Corrected: 10 th Oct 2015

Accepted for Publication: $24^{\text {th }}$ Nov 2015 attended orthopaedic outpatient department with complaints pain and decreased ROM of knee.

\section{Case Report}

A 31 years old gentleman who presented in orthopaedic outpatient department with complaints of knee pain for 2 week duration which was insidious in oneset,slowly progressive in nature,moderate in intensity and dull aching in character. He also complaints of difficulty in sitting cross legged,squatting and he also pointed to a swelling in the medial aspect of distal thigh. No history of trauma,fever,weight loss, decreased appetite or night cries.He was a non-vegetarian. On local examination, there was raised temperature, tenderness and indurations of vastus medialis muscle on the medial 
distal thigh. Knee effusion was also present. He had normal extension of knee but flexion was only upto 30 degrees.

Systemic examination was normal. Complete hemogram and infective markers including ESR and
CRP were normal. USG of thigh reported as muscle tear in the vastus medialis muscle and knee effussion.So patient was given gutter slab for immobilization of thigh and knee and NSAID was prescribed for pain relief.
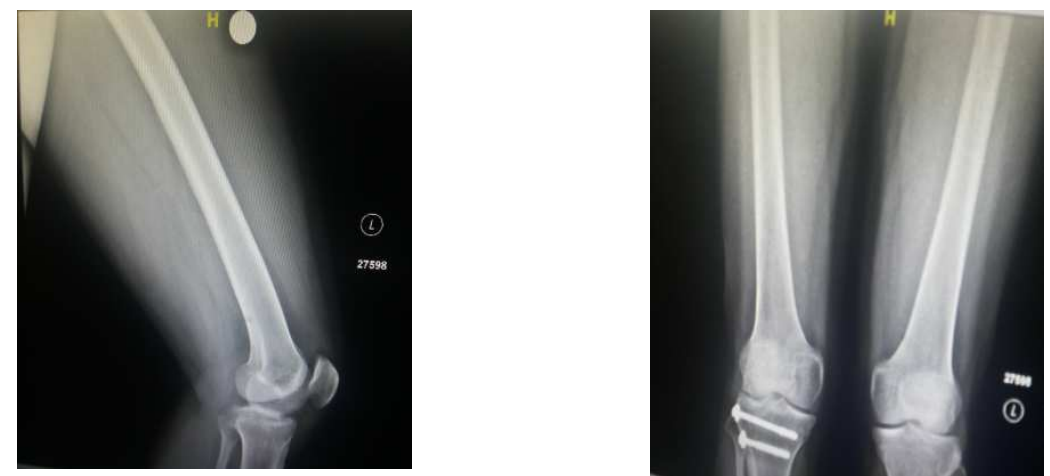

Figure 1: Lateral view of $X$ - ray knee Figure 2: AP view of $X$ - ray knee

After 3 days of immobilization patient was reviewed with severe complaints of knee pain and increased swelling of distal thigh and knee effusion. He was not able weight bear on the affected limb due to pain. At that point of time MRI was advised as the repeat ultra sound was not showing any cystic swelling or mass lesion except for small tear in the vastus medialis and knee effusion. MRI had shown $1 \times 0.8 \times 0.5 \mathrm{~cm}$ sized well defined oval cystic lesion in the deep muscular plane of vastus medialis in its distal one third segment. The lesion showedT2/PD hyper intense and T1 hypo intense signal intensities and small punctate hypo intensity noted within the lesion. There was thin T2 hypo intense peripheral rim present.T2/PD hyper intensities also noted in surrounding muscle fibres suggestive of edema. Rest of the visualized thigh muscles appeared normal. Left knee joint structures were normal except for knee joint effusion and it was diagnosed as intra muscular cysticercosis.
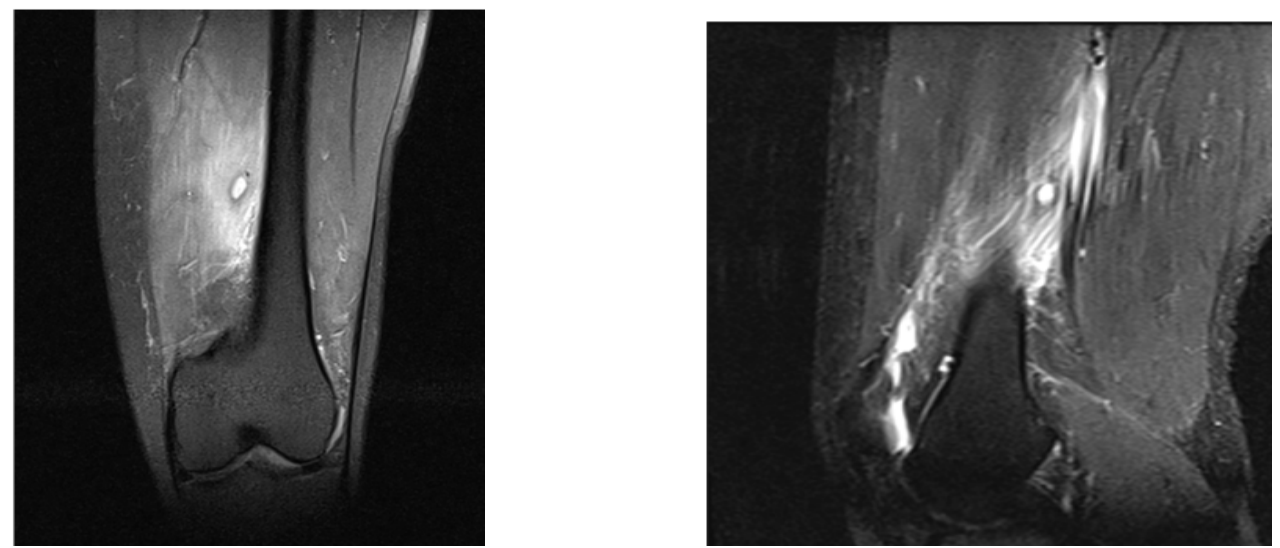

Figure 3 \& 4: Coronal \&Sagittal section of knee showing ring shaped lesion

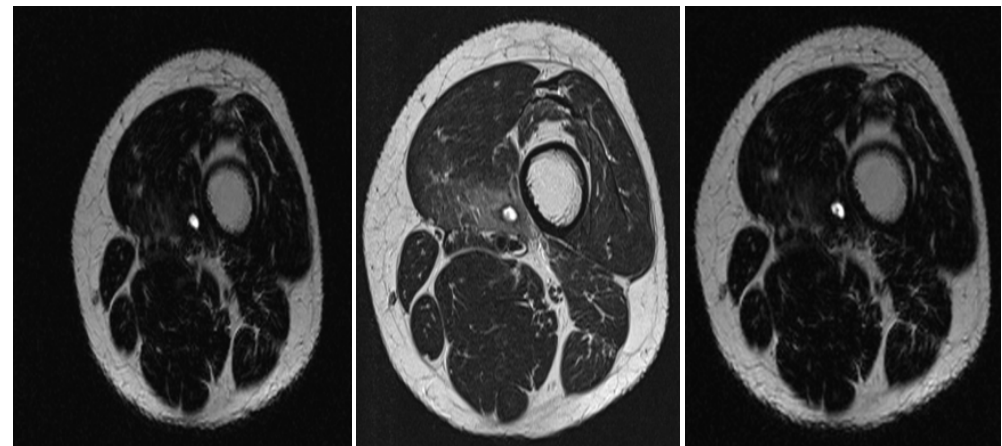

Figure 5,6\&7: axial section of distal thigh showing ring shaped lesion 
Serology for parasitic infection was done which showed higher titres of antibody to teania solium infection as shown below. Since single lesion cysticercosis is rare without central nervous system involvement MRI of brain was ordered, but it showed no focal enhancing lesion/cysts except few tiny non-specific T2 hyper intense foci in bilateral white matter.
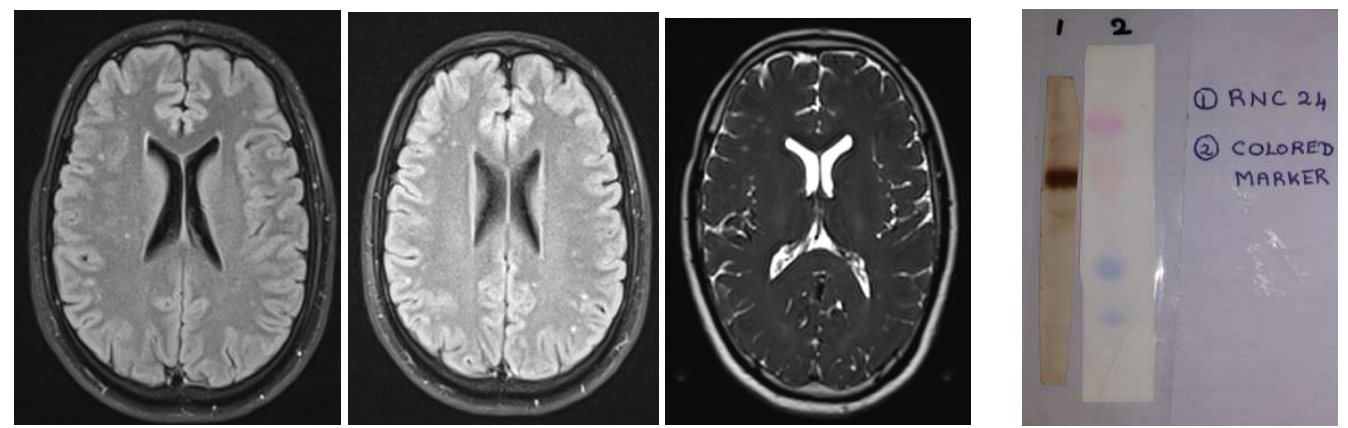

Figure 8, 9, 10: MRI images of brain as shownshowing no lesion Figure 11: positive serology

This patient was given tablet albendazole at a dose of $15 \mathrm{mg} / \mathrm{kg} /$ day for 28 days. After four weeks of drug therapy, he was completely asymptomatic with complete resolution of pain and full knee ROM was regained. Patient was followed up after 2 months with no complaints and a repeat MRI was done with no evidence of lesion in the thigh.

\section{Discussion}

Cysticercosis is caused by larval stage of pork tapeworm (Taenia solium) [1]. Cysticercosis is common in Africa, India,China and some part of America[7]. Tapeworm infestation is found to be common in under developed countries with poor sanitation and overcrowding causing higher chance of feco oral contamination of water with infective organism[3,15]. Pigs are the intermediate host and man is definitive host for the parasite taenia solium.The infection of humans starts with the use of water or food contaminated with eggs of infective agent. In the intestine oncospheres released from eggs which will penetrate mucosa of intestine and reach muscles/soft tissues causing cysticercuscellulosae (encysted stage of T. solium. Patient has been found to be asymptomatic in majority of cases of cysticercosuscellulosae .Symtpomaticcysticercosus has been typed into 3 which includes myalgic, tumour, and pseudotumour types $[1,3]$.

There are studies where high resolution sonography had been used to diagnose muscular cysticerocusis $[2,7]$. But in our study we were not able to come to a final conclusion based on ultrasound findings alone. It can be due to very small size of the lesion. We came to a diagnosis of cysticercosis base on the MRI findings. MRI had shown well defined oval cystic lesion in the deep muscular plane of vastus medialis with T2/PD hyper intense and $\mathrm{T} 1$ hypointense signal intensities and small punctate hypo intensity noted within the lesion(suggestive of scolex).T2/PD hyper intensities also noted in surrounding muscle fibers suggestive of edema of muscles. The swelling of knee and severe pain with features of inflammation is supposed to be due to death of the larva inside the cyst and breakage of cystic wall causing exudation of toxic materials to surrounding tissues leading to host immune reaction.

In this patient Complete hemogram showed no increase in esinophils even though he was symptomatic with pain, inflammation and effusion of knee. This is in contrast to some other case report where symptomatic helminthic infection is associated with raised esinophils $[10,16]$.Plain $x$-ray of knee with thigh showed no bony lesion or periosteal reactions and diagnosis of cysticerci by xray is not easy except in chronic cases. We were not able to diagnose cysticercosus by ultra sound may be because of very small size of the lesion in our case. In general it is really a challenge to diagnose cysticercosis by ultra sound since there is higher inter observer variation.

On the basis of serology and MRI findings, albendazole was started at a dose of $15 \mathrm{mg} / \mathrm{kg} /$ dose for 4 weeks (old regim) even if there are novel trials which showed good response with 8 day therapy with albendazole [4]. Antihelminthic drug albendazole acts by depleting ATP level and causing demise of parasite. Patient was symptomatically better in terms of pain after 1 week of drug therapy but complained of decreased flexion of knee with difficulty in squatting and sitting cross legged. Patient was completely asymptomatic with full 
range of knee ROM after treatment with albendazole alone for duration of 4 weeks.

\section{Conclusion}

We should keep Intra muscular cysticercosis as a differential diagnosis for knee pain especially in endemic areas. Muscular cysticercosus with no other systemic involvement can be treated with albendazole alone. Since isolated cysticercosus is a rare entity we should always rule out other systemic involvement especially central nervous system.

Funding: None, Competing interests: None declared Ethical clearance: The study was approved by the institutional human ethical committee.

\section{References}

1. Vijayaraghvan SB. Sonographic appearances in cysticercosis. J Ultrasound Med. 2004;23:423.

2. Sidhu R, Nada R, Palta A, Mohan H, Suri S. Maxillofacial cysticercosis: Uncommon appearance of a common disease. J Ultrasound Med 2002;21:199-202.

3. Mittal A, Das D, Iyer N, Nagaraj J, Gupta M. Masseter cysticercosis - a rare case diagnosed on ultrasound. DentomaxillofacRadiol. 2008 Feb;37(2):113-6. doi: 10.1259/dmfr/31885135.

4. Vanijanonta S. Cysticercosis by the Year 2000: an Update. The J tropical med parasit 1999; 22(1):34-40

5. Vorachai S, Suphaneewan J. An Intramuscular Cysticercosis, A Case Report with Correlation of Magnetic Resonance Imaging and Histopathology. Chot Mai Het ThanPhaet2007; 90(6): 1248-1252

6. Jankharia BG, Chavhan GB, Krishnan P et al. MRI and ultrasound in solitary muscular and soft tissue cysticercosis. Skeletal Radiol 2005; 34: 722-726

7. Asrani A, Morani A. Primary Sonographic Diagnosis of Disseminated Muscular Cysticercosis. J Ultrasound Med 2004; 23:1245-1248.
8. Khan RA, Chana RS. A Rare Cause of Solitary Abdominal Wall Lesion. Iran J paediatr 2008; 18(3): 291-292.

9. Ogilvie CM, Kasten P, Rovinsky D et al. Cysticercosis of the triceps: an unusual pseudotumor. ClinOrthop 2001; 382: 217-221

10. Gutierrez Y. Cysticercosis, Coenurosis, Sparganosis and Proliferating Cestode Larva. In: Diagnostic pathology of parasitic infections with clinical correlations, 2nd ed. Oxford University Press US 2000. p. 636-638

11. Mani NB, Kalra N, Jain M et al. Sonographic diagnosis of a solitary intramuscular cysticercal cyst. J Clin Ultrasound 2001; 29: 472-475.

12. Bilge EF, Baris $\mathrm{T}$, Ulku $\mathrm{K}$ et al. Solitary Cysticercosis in the Intermuscular Area of the Thigh: A Rare and Unusual Pseudotumor with Characteristic Imaging Findings [Case Report: Musculoskeletal Imaging]. Journal of Computer Assisted Tomography 2005; 29(2): 260-263.

13. Abdelwahab IF, Klein MJ, Hermann $G$ et al. Solitary cysticercosis of the biceps brachii in a vegetarian: a rare and unusual pseudotumor. Skeletal Radiol 2003; 32: 424-428

14. Brown ST, Brown AE, Flipa DA et al. Extraneural cysticercosis presenting as a tumour in a seronegative patient. Clininf dis1992; 14:53-558.

15. Ergen FB, Turkbey B, Kerimoglu U, Karaman K, Yorganc K, Saglam A. Solitary cysticercosis in the intermuscular area of the thigh: a rare and unusual pseudo tumor with characteristic imaging findings. J Comput Assist Tomogr. 2005;29(2):260-263.

16. Falco OB, Pleweig G, Wolf $\mathrm{HH}$ et al. Diseases caused by worms. In: Dermatology, Falco OB, Plewig G, Wolff HH, Winkelmann RK Eds. 3rd ed. Berlin, Springer-Verlag; 1984. p. 262-274), Pp:291-292.

\section{How to cite this article?}

Ali Mohammed P, Jagdish Menon, DK Patro, Deep Sharma. Cystiysticercosis as a differential diagnosis of knee pain: A rare case report. Int J Med Res Rev 2015;3(10):1269-1272. doi: 10.17511/ijmrr.2015.i10.230. 